\title{
Adult neurogenesis workshop report
}

\section{Shira Rosenzweig* and Paul Luu}

Department of Physiology, University of Toronto, Toronto, ON, Canada

*Correspondence: s.rosenzweig@utoronto.ca
The workshop was held on July 3, 2009, at the University of Toronto, Canada, and provided a forum for researchers in the field of adult neurogenesis to learn about recent progress in the field, and to present and discuss their ongoing research. The program included talks by mostly local trainees and faculty from the field of adult neurogenesis, who presented new data and reflected on emerging research directions. The presented topics encompassed the physiological roles that young neurons play in adult brain, as well as new venues in stem cells research and their therapeutic potential.

The organizer, Dr. Martin Wojtowicz from the Department of Physiology, opened the workshop and presented recent data pertaining to one of the central questions in the field: What is the functional significance of neurogenesis to learning and memory? Numerous studies have been attempting to answer this question in recent years, often with contradicting results. Dr. Wojtowicz suggested a novel role for adult neurogenesis in memory interference, and explained that the transient nature of young neurons may contribute to their function in this model; memories are encoded during a specific developmental stage in which the cells respond to certain stimuli, and once the cells develop beyond that stage, the encoded memories become "protected" from interference. Dr. Wojtowicz presented new supporting data, showing that adult rats in which neurogenesis in the dentate gyrus (DG) was decreased by irradiation, performed poorly in a task involving high interference when compared to non-irradiated rat. As a final note, Dr. Wojtowicz emphasized the importance of proper animal model selection in the study of adult neurogenesis, seeing as a large variability in the levels of neurogenesis exists across species.

Dr. Jason Snyder, a postdoctoral fellow in Dr. H. Cameron laboratory at NIH, Bethesda, USA, shared some of his recent findings in a presentation titled "Adult-born hippocampal neurons are more numerous, faster maturing and more involved in behavior in rats than in mice.” Dr. Snyder suggested that conflicting findings in the field stem from significant variations in neurogenesis that exist between mice and rats, and that many of the controversies result from researchers incorrectly comparing data between the two species. Dr. Snyder outlined a comparative study that evaluated different aspects of adult neurogenesis in rats and compared them to mice. By assessing the co-expression of BrdU and NeuN in adult-born DG neurons, Dr. Snyder demonstrated that these neurons mature faster in rats than in mice. Further staining for immediate-early genes, which are presumably expressed only in functional neurons that successfully integrated into the hippocampal circuitry, suggested that adult-born neurons in rats become functional approximately 2 weeks earlier than in mice. Long-term neuronal survival studies using BrdU also demonstrated that the number of adult-born neurons surviving to 6 weeks is greater in rats than in mice. In addition, Dr. Snyder presented data showing performance deficits in the contextual fear conditioning task following irradiation in rats, but not in mice, concluding that the contribution of young neurons to learning and memory is more pronounced in rats than in mice.

Ms. Nadia Sachewsky, a graduate student in the laboratory of Dr. C. M. Morshead in the Department of Anatomy and Cell Biology, shared some of her recent work in a presentation titled "The mother of all neurons: Neural stem cells and how to keep them happy." Ms. Sachewsky's research focuses on neural stem cells in the subependyma lining the lateral ventricles, and attempts to characterize factors that regulate their survival, specifically stromal cell-derived factor-1 (SDF-1) and stem cell factor (SCF). Ms. Sachewsky demonstrated that an infusion of these factors into the ventricle resulted in increased survival of subependymal stem cells, which has implications for neural tissue regeneration following stroke. She pointed out that the stem cell population decreases with age, potentially limiting regeneration, however, the use of SDF-1 and SCF provides promise in enhancing regeneration in aged neural stem cells.

Dr. Masashi Fujitani, a postdoctoral fellow in Dr. F. D. Miller's laboratory at the Department of Physiology, shared his recent findings in a presentation titled "TAp73 regulates the self-renewal and maintenance of CNS neural stem cells." Dr. Fujitani researches the molecular basis behind the ability of neural precursor cells to self-renew, and has identified a specific gene, TAp73 (p53 tumor suppressor gene homolog), which plays an important role in this process. Neurosphere assay and immunohistological techniques demonstrated that in hippocampal cultures prepared from TAp73 knockout mice, the self-renewal and proliferation of neuronal precursor cells are reduced. In subsequent experiments TAp73 was knocked down in utero, resulting in a similar decrease in proliferation. Dr. Fujitani then demonstrated that TAp73 also plays a role in adult neurogenesis. When comparing hippocampal sections stained for BrdU and NeuN, he found a decrease in the number of double-stained cells in TAp73 knockout mice compared to control mice. Using microarray analysis Dr. Fujitani had successfully identified Hey2 promoter as a possible downstream target of TAp73.

Dr. Tzong-Shiue Yu, a postdoctoral fellow in Dr. P. W. Frankland's laboratory at the Department of Physiology, outlined his recent findings in a presentation titled "Neuronal Activity in Entorhinal Cortex Regulates Adult Neurogenesis". Dr. Yu's work examines whether exposure to enriched environment promotes neurogenesis by increasing the inputs relayed to the DG from the entorhinal cortex. Dr. Yu used viral transfection to transiently over-express either dominant-negative KCNQ2 or Kir 2.1 in the entorhinal cortex of rats (the primary input to the DG), thus increasing or decreasing the excitability in this region, respectively. Preliminary results suggested that increased excitability of the entorhinal cortex, which would increase the inputs into the DG (possibly in a similar way to 
the increase caused by exposure to enriched environment) resulted in an increase in neuronal proliferation and differentiation in the DG.

Dr. Scellig Stone, a graduate student in Dr. P. W. Frankland's laboratory in the Department of Physiology and Dr. A. Lozano's laboratory in the Department of Surgery, outlined his work in a presentation titled "Integration of DG neurons into memory circuits: Does age matter?" Dr. Stone's research explores whether adult-born neurons possess similar ability to integrate into existing hippocampal networks and functionally contribute to behavior as those born during development. As a first step, Dr. Stone injected animals with BrdU and trained them in the Morris water maze in order to induce the expression of immediate-early genes (such as fos). By varying the time between injection and training, Dr. Stone demonstrated that adult-born DG neurons become integrated and functional (as determined by fos expression) at approximately 5 weeks of age. Dr. Stone then used two proliferation markers: CldU and IdU, to label two distinct populations of neurons in the DG - one born in early postnatal life (P7), and the other during adulthood (P60). The rats were then trained on a contextual fear conditioning task to induce fos expression. The results revealed that the fraction of CldU-positive cells (born during development) that expressed fos was similar to the fraction of IdU-positive cells (born in adulthood) that expressed fos. These results suggest that both populations of neurons integrate into the DG circuitry at similar rates. The finding that adult-born neurons are functionality interchangeable with neurons born during development could reinforce the notion that these cells may be utilized in future therapeutic strategies of regenerative medicine.

Dr. Sabrina Wang from NIH, Taipei, Taiwan, shared her work in a presentation titled "Learned helplessness model of depression and hippocampal neurogenesis." Dr. Wang's research focuses on depression, a complex condition of growing concern among worldwide health organizations.
Two rat models for depression were used by Dr. Wang. In the first, rats were exposed to chronic mild stress, and the level of "depression" was assessed by the sucrose consumption test or forced swim test. In the second "learned helplessness" model, rats were administered inescapable electrical shocks, and at a later point presented with an "escape route" (avoidance test). Rats that chose not to avoid the shocks were considered "depressed" (LH). When studying the DG in these rats, Dr. Wang found a significant decrease in neurogenesis as assessed by BrdU and doublecortin positive cells, in the dorsal hippocampus of LH rats compared to controls, suggesting a link between stress, depressive behavior and neurogenesis in these animals.

Dr. Mary Pat McAndrews, from the Department of Psychology, University of Toronto, offered some of her recent findings in a presentation titled "Memory effects of low dose brain irradiation in humans." Dr. McAndrews presented novel data which further supports a connection between neurogenesis and behavior in humans. In her study, human patients who've undergone treatment with a gamma knife for various conditions were tested in several behavioral paradigms aimed at assessing relational memory processing. The gamma knife treatments had collateral exposure sites on the hippocampus, thus exposing the DG to levels of radiation that are comparable to those used experimentally to reduce neurogenesis in laboratory animals. Preliminary results from the memory tests suggested a transient impairment in specific types of relational memory processing 1 month after the gamma knife treatment, with some correlation between the change in performance and the dose of radiation. These impairments disappeared completely 3 months after the treatment.

The last talk in the workshop was given by Dr. Sue Becker from McMaster University, who shared new data in a presentation titled "Running, depression and neurogenesis in humans.” Dr. Becker's past work focused on the connection between neurogenesis and depression in humans. Her lab tested a large number of undergraduate volunteers in the stress and depression test, specifically trying to identify students who were in a first bout of depression. The students were then tested in several memory tasks, some involving high interference. Because of the reported connection between depression and reduced neurogenesis, Dr. Becker expected to find impairment in memory processes that require neurogenesis in depressed students. Indeed, their performance on the delayed match to sample task was significantly impaired in agreement with the hypothesis.

Mr. Nick Dery, a graduate student in Dr. Becker's laboratory, continued the presentation. His research involves the use of an imaging technique utilizing MRI to measure cerebral blood volume. Mr. Dery described a previously shown correlation between blood flow to the DG and the levels of adult neurogenesis, and proposed that measuring cerebral blood volume in the DG can be used as a non-invasive method of estimating the rate of adult neurogenesis in humans. Referring to the common finding that running increases neurogenesis in rodents, Mr. Dery expects to find an increase in cerebral blood volume in the DG of volunteers who undergo a physical exercise routine, and hopes that this increase would also manifest as improved performance in memory tasks that are postulated to involve adult neurogenesis.

The workshop was sponsored by BRAIN platform, Department of Physiology, and the University of Toronto Neuroscience Program.

Conflict of Interest Statement: The authors declare that the research was conducted in the absence of any commercial or financial relationships that could be construed as a potential conflict of interest.

Published online: 23 August 2010.

Citation: Rosenzweig S and Luu P (2010) Adult neurogenesis workshop report. Front. Neurosci. 4:57. doi: 10.3389/ fnins.2010.00057

This article was submitted to Frontiers in Neurogenesis, a specialty of Frontiers in Neuroscience.

Copyright $\odot 2010$ Rosenzweig and Luu. This is an open-access article subject to an exclusive license agreement between the authors and the Frontiers Research Foundation, which permits unrestricted use, distribution, and reproduction in any medium, provided the original authors and source are credited. 